\title{
The oncolytic effect in vivo of reovirus on tumour cells that have survived reovirus cell killing in vitro
}

\author{
T Alain', M Kim', RN Johnston', S Urbanski ${ }^{2}$, AE Kossakowska ${ }^{1,2, *}$, PA Forsyth ${ }^{1,4}$ and PWK Lee , $^{*, 3,4}$ \\ 'Department of Medical Sciences, University of Calgary, Calgary, Alberta, Canada; ${ }^{2}$ Department of Pathology, University of Calgary, Calgary, Alberta, \\ Canada; ${ }^{3}$ Department of Microbiology and Immunology, Dalhousie University, 7/F Sir Charles Tupper Building, 5850 College Street, Halifax, Nova Scotia, \\ Canada B3H IX5
}

\begin{abstract}
The use of oncolytic viruses has received considerable attention in recent years and many viruses have proved to be effective against a variety of cancer models and a few are currently being used in clinical trials. However, the possible emergence and outcome of virus-resistant tumour cells has not been addressed. We previously reported the effective use of reovirus against lymphoid malignancies, including the Burkitt's lymphoma cell line Raji. Here we isolated in vitro persistently infected (PI) Raji cells, and cells 'cured' of persistent reovirus infection ('cured' cells). Both PI and cured Raji cells resisted reovirus infection and cell killing in vitro. In vivo, the PI cells were non-tumorigenic in SCID mice, but cured cells regained the parental cells' ability to form tumours. Tumour xenografts from the cured cells, however, were highly susceptible to reovirus oncolysis in vivo. This susceptibility was due to the proteolytic environment within tumours that facilitates reovirus infection and cell killing. Our results show that persistent infection by reovirus impedes tumour development and that although PI cells cleared of reovirus are tumorigenic, they are killed upon rechallenge with reovirus. Both the $\mathrm{PI}$ and cured states are therefore not likely to be significant barriers to reovirus oncolytic therapy. British Journal of Cancer (2006) 95, I020- 1027. doi:I0.1038/sj.bjc.6603363 www.bjcancer.com (c) 2006 Cancer Research UK
\end{abstract}

Keywords: reovirus oncolysis; persistent infection; cured cells; tumour regression

Reovirus is the prototype of the Reoviridae family of doublestranded RNA viruses that is found ubiquitously in the environment. Although most humans have been infected with this virus, there is little or no manifestation of clinical symptoms (Tyler and Fields, 1996). The virus is not linked to any known human diseases and is therefore considered to be benign. More recently however, reovirus has been shown to be an effective oncolytic agent against a variety of cancers (Wilcox et al, 2001; Hirasawa et al, 2002; Norman et al, 2002; Etoh et al, 2003; Kilani et al, 2003) including lymphomas (Alain et al, 2002). These studies led to clinical trials of reovirus as a cancer therapeutic (Morris et al, 2002).

While the use of viruses as oncolytic agents has shown considerable promise in preclinical studies (Kirn, 2002; Bell et al, 2003), the possibility of generating or selecting for cancer cells that have become resistant to reovirus has not been evaluated. It is possible that a subpopulation of cancer cells may survive the initial viral assault and become persistently infected (PI) with the virus. Although it is not known if such residual cancer cells actually exist in vivo, the fact that cells PI with reovirus can be established in vitro (Taber et al, 1976; Ahmed et al, 1981; Ahmed and Fields, 1982; Danis et al, 1993; Dermody et al, 1993, 1995; Dermody, 1998) suggests that this could be a potential problem in vivo. It is therefore important to evaluate the tumorigenicity of such PI cells

*Correspondence: Dr PWK Lee; E-mail: patrick.lee@dal.ca

* Deceased March 2003

${ }^{4}$ Shared senior authorship

Revised II August 2006; accepted 17 August 2006 relative to that of the parental cells to see if this could represent a mechanism for proliferating tumour cells to escape initial viral oncolysis.

Another more important concern pertains to the potential generation, from the PI cells, of cells 'cured' of viral infection. In the case of reovirus, cured cells can be generated from PI murine L cells simply by incubation with anti-reovirus antibodies. Importantly, these cells then lack permissiveness to wild-type reovirus in vitro (Ahmed et al, 1981; Dermody et al, 1993; Baer and Dermody, 1997; Wetzel et al, 1997; Baer et al, 1999). Whereas the presence of viruses in PI cells may keep tumour cells in check, cured cells without such a deterrent could potentially develop into tumours. If cured tumours are now resistant to the original wild-type virus, this may pose another potential therapeutic challenge.

In this study, we addressed these issues using the Burkitt's lymphoma cell line Raji, which we have found to be highly susceptible to reovirus oncolysis (Alain et al, 2002). Despite this exquisite susceptibility in vitro, we isolated a resistant population that was PI, and generated cured cells from this PI population using anti-reovirus antibodies. We compared the PI and cured cells to the parental cells in terms of tumorigenicity and susceptibility to reovirus oncolysis in vitro and in vivo. We found that PI Raji cells were nontumorigenic, but cured cells formed large tumours in vivo. Raji cured cells were resistant to reovirus infection in vitro, but were highly susceptible to reovirus oncolysis in vivo. Subsequent experiments demonstrated that the presence of proteases within the microenvironment of Raji cured tumours enhanced the processing and hence the oncolytic activity of reovirus in vivo. In view of the lack of tumorigenicity of the PI cells 
and the susceptibility of cured cells to reovirus in vivo, we conclude that these conditions should not represent a significant barrier for reovirus oncolytic therapy.

\section{MATERIALS AND METHODS}

\section{Cells and reovirus}

The human Burkitt's lymphoma cell line Raji, obtained from American Type Culture Collection (ATCC), was maintained in RPMI containing 10\% foetal bovine serum (FBS), 1\% L-glutamine (Gibco/BRL, Burlington, ON, Canada) and antibiotics (SigmaAldrich, St Louis, MO, USA). Cells were kept at $37^{\circ} \mathrm{C}$ in a humidified $5 \% \mathrm{CO}_{2}$ incubator. For the growth curve assay, Trypan Blue exclusion test was performed every $24 \mathrm{~h}$. The cells were stained with $0.25 \%$ dye and viable cells in three independent wells were counted using a haemocytometer.

The PI cells were established after infection of the parental Raji cells with reovirus type 3 Dearing and isolating the surviving populations. The surviving cells were subsequently re-infected three times a week for 3 weeks at 20 plaque-forming units (PFU) per cell to eliminate all cells susceptible to lysis. The cells then underwent two periods of crisis in which the majority of cells died, but resistant cells eventually emerged and remained PI (Raji PI). Raji cured cells were generated by culturing the PI cells in the presence of $1 \%(\mathrm{v} / \mathrm{v})$ filter sterilised polyclonal rabbit anti-reovirus serotype 3 antiserum. Cells were passaged in the presence of antiserum every second day for a period of 2 weeks. The cell population obtained following this treatment contained no detectable viral particles.

The Dearing strain of reovirus serotype 3 was propagated in L929 cells grown in suspension in Joklik's modified Eagle's medium (JMEM) containing 5\% FBS. The virus was purified according to the protocol of Smith et al (1969), with the exception that $\beta$-mercaptoethanol was omitted from the extraction buffer.

Infectious subviral particles (ISVPs) were obtained by digesting virions $\left(1 \times 10^{11} \mathrm{PFU} \mathrm{ml}{ }^{-1}\right)$ with $N$ - $\alpha$ - $\rho$-tosyl-t-lysine chloromethyl ketone (TLCK)-treated $\alpha$-chymotrypsin $\left(200 \mu \mathrm{g} \mathrm{ml}^{-1}\right)$ (SigmaAldrich) for $30 \mathrm{~min}$ at $37^{\circ} \mathrm{C}$. Digestion was stopped by the addition of ethanolic phenymethysulphonyl fluoride (5 mM) (Sigma-Aldrich) at $4{ }^{\circ} \mathrm{C}$, and the ISVPs generated were used immediately for cell infection.

\section{Polymerase chain reaction and immunofluorescence}

Cells were harvested and RNA was extracted using the RNeasy protocol (Qiagen Inc., Missassauga, ON, Canada). Equal amounts of total cellular RNA from each sample were then subjected to RT-PCR as described previously (Strong et al, 1998). S1 cDNA primers: 5'-AATTCGAATTAGGTGACACTATAG-CTATTGGTCG GATG- $3^{\prime}$ and $5^{\prime}$-CCCTTTTGACAGTGATGCTCCGTTATCACTCG$3^{\prime}$. S2 cDNA primers: $5^{\prime}$-TTCGCTGGTCAGTTATGGCTC- $3^{\prime}$ and 5'-TACCAGCTCGGAGTAGATGTG-3'.

For immunofluorescent studies, cells were spread and fixed on slides using Cytology Fixative (Surgipath, Richmond, IL, USA). After rehydration by sequential washes in 75,50 and $25 \%$ ethanol followed by four washes with phosphate-buffered saline (PBS), the fixed and rehydrated cells were treated with $10 \%$ goat serum in PBS and then exposed to the primary antibody (rabbit polyclonal anti-reovirus type 3 serum diluted $1 / 5000$ in PBS) overnight at $4^{\circ} \mathrm{C}$. Following three washes with PBS, the cells were exposed to the secondary antibody (fluorescein isothiocyanate (FITC)-conjugated goat anti-rabbit IgG diluted $1 / 100$ in PBS) for $1 \mathrm{~h}$ at room temperature. The slides were then washed three times with PBS, mounted with DAPI mounting medium (Vector Laboratories, Burlingame, CA, USA), and the cells were viewed and photographed with a Zeiss microscope (magnification $\times 400$ ).

\section{Ras activation assays and Western blot analysis}

Ras-GTP levels were measured using a Ras activation assay kit (Upstate Biotechnology, Lake Placid, NY, USA). Subconfluent cells in $15-\mathrm{cm}$ dishes were washed two times in ice-cold TBS and lysed by scraping in $500 \mu \mathrm{l}$ of $\mathrm{Mg}^{2+}$ lysis buffer (MLB, Upstate Biotechnology). Six hundred microlitres of precleared lysate was then incubated with $30 \mu \mathrm{l}$ of GST - Raf Ras-binding domain bound to glutathione agarose for $45 \mathrm{~min}$ at $4{ }^{\circ} \mathrm{C}$. Beads were then washed three times in MLB and boiled in $40 \mu \mathrm{l}$ of $2 \times$ protein sample buffer with $2 \mu \mathrm{l}$ of $1 \mathrm{M}$ DTT. Twenty microlitres of pull-down sample and $40 \mu \mathrm{l}$ of total lysate were then subjected to $10 \%$ SDS-PAGE and transferred to nitrocellulose membrane. Blots were probed overnight with 1000-fold diluted RAS10 mAb (Upstate Biotechnology) and then washed three times for $5 \mathrm{~min}$ in TBS $/ 0.1 \%$ Tween-20, followed by incubation in goat anti-mouse-HRP Ab $(1: 2500$, Santa Cruz Biotechnology, Santa Cruz, CA, USA). After three more washes, blots were processed and signals were visualised using enhanced chemiluminescence (Amersham Biosciences, Little Chalfont Buckinghamshire, England).

For detection of activated extracellular signal-regulated kinase (ERK), cells in complete medium were washed in ice-cold TBS and lysed in standard radioimmunoprecipitation assay buffer with phosphatase inhibitors (TBS, $0.1 \%$ SDS, $0.5 \%$ sodium deoxycholate, $1 \%$ Triton $\mathrm{X}-100,10 \mathrm{~mm}$ sodium pyrophosphate, $25 \mathrm{~mm}$ $\beta$-glycerophosphate, $1 \mathrm{~mm}$, sodium orthovanadate, $25 \mathrm{~mm}$ sodium fluoride). Forty microlitres of precleared lysate were then analysed by Western blot using the anti-phospho-ERK1/2 and anti-total ERK1/2 Abs obtained from Cell Signaling Technology (Beverly, MA, USA).

\section{Soft agar assay}

A total of $1 \times 10^{5}$ cells of parental, PI and cured cells were mixed $(1: 1)$ in $2 \times$ RPMI containing $10 \%$ FBS and $1.2 \%$ low-melting temperature agarose (SeaPlaque, Rockland, ME, USA) and allowed to grow for 4 weeks. Colonies were then fixed in ethanol/acetic acid overnight and stained with Coomassie brilliant blue. Photomicrographs of the wells were taken on a Kodak DC290 ZOOM digital camera.

\section{Reovirus infection of the Raji cell lines in vitro}

One million cells of each cell line were dispensed into 24-well plates and infected with reovirus or ISVPs at an MOI of $20 \mathrm{PFU} \mathrm{cell}{ }^{-1}$. For experiments involving the use of proteases, infection with reovirus was carried out in the presence of $10 \mu \mathrm{g} \mathrm{ml}^{-1}$ TLCK-treated chymotrypsin (replaced every $24 \mathrm{~h}$ for up to $96 \mathrm{~h}$ ). For E64 protease inhibitor (Sigma) treatment in vitro, cells were exposed to $50 \mu \mathrm{M}$ of E64 in PBS $1 \mathrm{~h}$ before reovirus infection. Virus was allowed to bind for $45 \mathrm{~min}$ at $4{ }^{\circ} \mathrm{C}$, after which E64 was again added to the cells to a final concentration of $50 \mu \mathrm{M}$.

WST-1 (4-[3-(4-iodophenyl)-2-(4-nitrophenyl)-2H-5-tetrazolio]-1,3-benzene disulphonate) assays (Roche, Diagnostics, Laval, Qc, Canada) were used to measure cell viability. Cells were infected as above and a tetrazolium salt WST-1 was added to the cultures for $6 \mathrm{~h}$ before the $96 \mathrm{~h}$ time point postinfection. The cleavage of WST-1 to formazan by metabolically active cells was quantified by scanning the plates at $450 \mathrm{~nm}$ reference wavelength in a microtitre plate reader. Medium without WST-1 was used as background control. The experiments were performed in triplicate.

For metabolic labelling, $\left[{ }^{35} \mathrm{~S}\right]$ methionine was added to the culture medium at designated times for a period of $6 \mathrm{~h}$. Cells were harvested and lysed in lysis buffer (PBS) containing 1\% Triton $\mathrm{X}-100,0.5 \%$ sodium deoxycholate and $1 \mathrm{~mm}$ EDTA). Lysates were cleared of debris by centrifugation and supernatants were stored at $-70^{\circ} \mathrm{C}$ until use. Polyclonal rabbit anti-reovirus serotype 3 serum was used for immunoprecipitation of $\left[{ }^{35} \mathrm{~S}\right]$ methionine-labelled 
reovirus proteins from cell lysates as described previously (Lee et al, 1981). Immunoprecipitated proteins were subjected to SDS PAGE (Laemmli, 1970) followed by autoradiography.

\section{Progeny virus production}

Three million cells grown in six-well plates were infected with reovirus at an MOI of 20 . At $96 \mathrm{~h}$ postinfection, the plates were frozen and stored at $-70^{\circ} \mathrm{C}$ until use. To assay for progeny virus production, the plates were subjected to three rounds of freezethaw, and the supernatants were used for plaque titration on L929 cells. All titration experiments were repeated in triplicate.

\section{SCID mice studies}

Six- to 8-week-old Fox-Chase SCID mice were obtained from the Jackson Laboratory (Bar Harbor, ME, USA). The animals were maintained under specific pathogen-free conditions and according to a protocol approved by the University of Calgary Animal Care Committee. As a xenograft model, $1.0 \times 10^{7}$ Raji or Raji-derived cells (PI or Cured) were injected subcutaneously in the hind flank of the mice. Once palpable tumours were established (day 0 ), $1.0 \times 10^{7} \mathrm{PFU}$ of live reovirus in PBS was administered intratumorally (experimental group), or PBS alone (control group). Twodimensional tumour measurements were performed with calipers every other day for 25 days or until the animals showed severe morbidity due to excess tumour burden or complications due to viral infection.

In vivo experiments involving the use of the protease inhibitor E64 were similarly carried out, with the exception that $1 \mathrm{mg}$ of E64 in PBS was injected IP into the mouse and subsequently every second day, until day 8 for the E64 alone and the E64+ reovirus group. This concentration was chosen from a previous report showing the inactivation of proteases by E64 in vivo without affecting the tumour growth of a Burkitt lymphoma tumour (Sebti et al, 1991). For the E64+ reovirus group, E64 injection was performed $1 \mathrm{~h}$ before the single reovirus injection. For ISVP treatment, ISVPs were prepared as described above and $1.0 \times 10^{7}$ PFUs were administered once intratumorally.

\section{Histology and immunohistochemistry}

Tumours (or remaining masses) taken from animals on day 20 post-intratumoral reovirus (or saline) injection were fixed in $10 \%$ neutral-buffered formalin and embedded in paraffin for histological analysis. Sections were then immersed in xylene, followed by rehydration in decreasing concentrations of ethanol. For histopathological examination, the sections were stained with haematoxylin and eosin (H\&E). For immunohistochemistry, endogenous peroxidase was inactivated in 3\% hydrogen peroxide in methanol for $15 \mathrm{~min}$. Sections were then incubated with a primary rabbit anti-reovirus polyclonal antibody (1/1000 in PBS with $10 \%$ goat serum and $0.1 \%$ Triton $\mathrm{X}-100$ ) that was partially purified by ammonium sulphate precipitation. Slides were washed in PBS and then subjected to avidin-biotin-horseradish peroxidase staining as recommended by the manufacturer (Vector, Burlingame, CA, USA) and counter-stained in haematoxylin.

\section{Statistical analysis}

Statistical Analysis Software GraphPad Prism (version 4, GraphPad Software Inc., San Diego, CA, USA) was used for statistical analyses. Wilcoxon's signed-rank tests were used to compare the distributions of tumour sizes. All reported $P$-values were two-sided and were considered to be statistically significant at $<0.05$.

\section{RESULTS}

\section{Growth of PI, cured and parental Raji cells in vitro}

To generate PI cells, parental Raji cells were subjected to multiple rounds of infection by reovirus type 3 Dearing. Most cells were killed by the virus, but a very small number of cells survived and were propagated for the next several months. After undergoing two crisis periods, a resistant population was established that was PI and that continuously shed infectious viral particles into the culture medium. These cells (designated Raji PI) could subsequently be cured of reovirus infection using anti-reovirus antibodies. Cured cells (designated Raji cured) contained no detectable viral proteins or RNA (see below). Evaluation of the cluster of differentiation antigens present on PI and cured cells verified their common origin from Raji cells (data not shown).

We first characterised and compared the Raji PI and Raji cured cells to the parental Raji cells. RT - PCR and immunofluorescence confirmed the presence of reovirus only in the PI cells (Figure 1A);
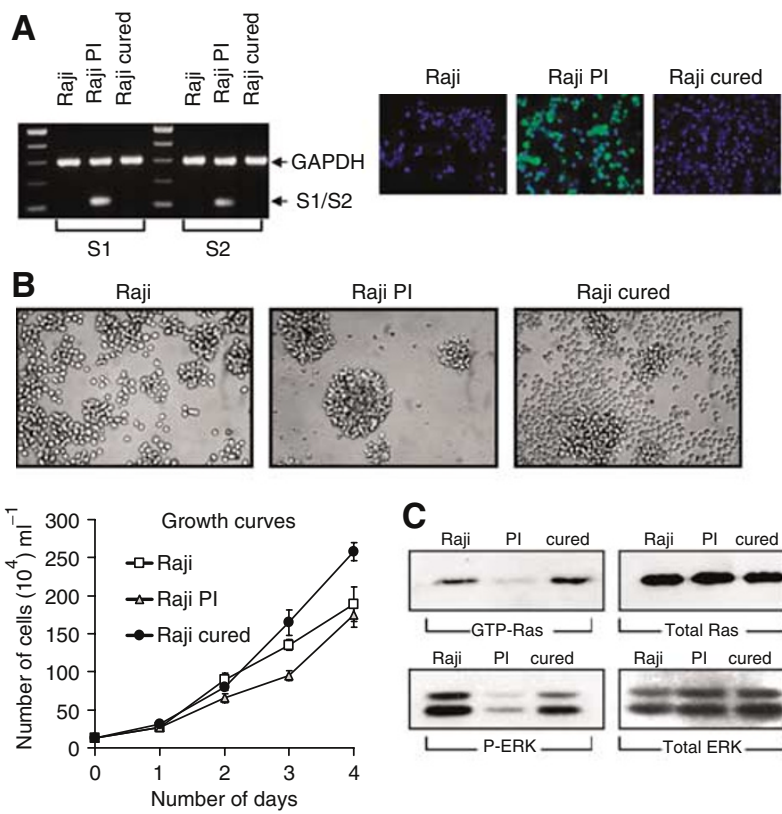

Raji PI

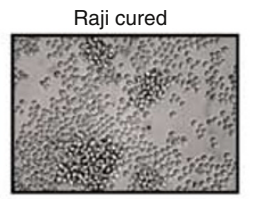

D Raj

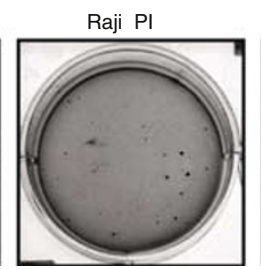

C
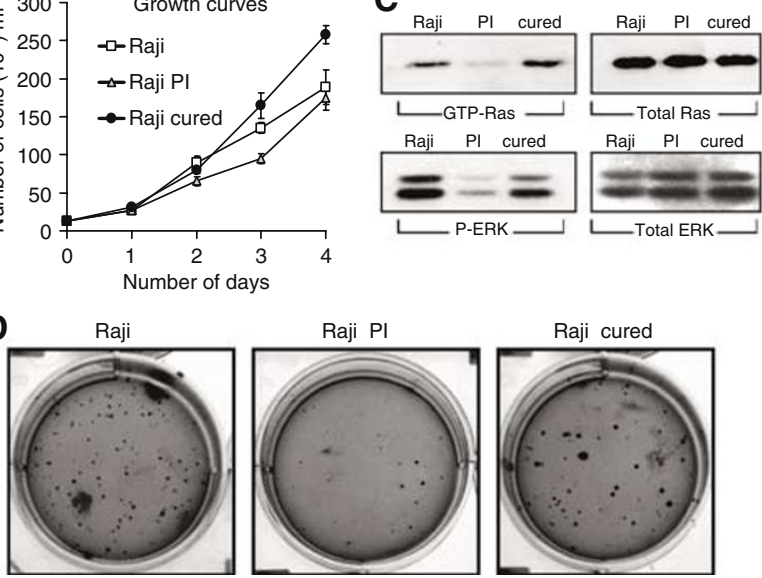

Raji cured

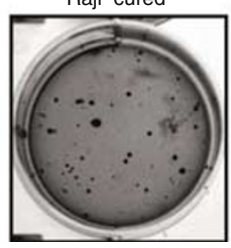

Figure I Growth of the Raji parental, PI and cured cells. (A) The left panel shows polymerase chain reaction of reovirus SI and S2 mRNA transcript in Raji parental, PI and cured cells. Equal amounts of RNA from each sample were subjected to RT-PCR, followed by selective amplification of reovirus SI or S2 cDNA and GAPDH. The right panel shows immunofluorescence of reovirus proteins expressed only in PI cells. Cells grown under normal conditions were fixed, processed and reacted with rabbit anti-reovirus type 3 antibody, followed by FITC-conjugated goat anti-rabbit IgG and mounted with DAPI-stained mounting medium. The magnification for all panels was $\times 400$. (B) Photomicrographs of the Raji parental, $\mathrm{Pl}$ and cured cells in culture and growth curves assessed by staining the cells with $0.25 \%$ Trypan Blue. Viable (unstained) cells from three independent wells were counted using a haemocytometer. (C) Ras and ERK activity in Raji, PI and cured cells. (D) Growth of Raji parental, PI and cured cells in soft agar. A total of $1 \times 10^{5}$ cells were mixed $(1: 1)$ in $2 \times \mathrm{RPMI}$ containing $10 \%$ FBS and $1.2 \%$ low-melting temperature agarose (SeaPlaque) and allowed to grow for 4 weeks. Colonies were then fixed, stained with Coomassie brilliant blue and photomicrographed. 
no viral transcripts or proteins were detectable in Raji cured cells. Persistently infected cells also tended to form clusters in culture and grew at a slightly slower rate while the proliferation rate of the cured cells surpassed that of the parental cells (Figure 1B). Examination of activated Ras-GTP and phosphorylated ERK1/2 levels revealed that both were significantly reduced in PI cells, but were restored in the cured cells to those seen for the parental cells (Figure 1C). Moreover, cured and parental Raji cells readily formed colonies in soft agar, whereas PI cells did not (Figure 1D); this suggests that the cured cells, like the parental cells, are likely tumorigenic whereas the PI cells are likely nontumorigenic.

\section{Parental and cured cells, but not PI cells, develop large tumours in vivo}

Despite numerous studies on reovirus PI cells, the tumorigenicity of PI and cured cells has never been reported. Accordingly, the cells were introduced subcutaneously into SCID mice, which had previously been shown to support the growth of Raji tumours. The results (Figure 2A) show that, in agreement with the in vitro soft agar assay, both the parental and cured cells produced large tumours, whereas Raji PI cells failed to grow in these mice. Histological examination showed large and clearly proliferating tumours in the Raji parental and Raji cured groups. The PI group had small tumours that could not be palpated and were detectable only microscopically by immunohistochemistry using anti-reovirus antibodies (Figure $2 \mathrm{~B}$ ). It is interesting to note that the
A

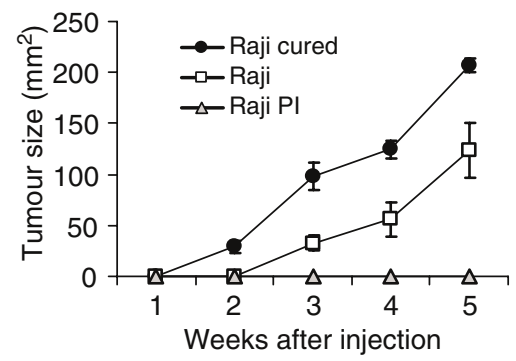

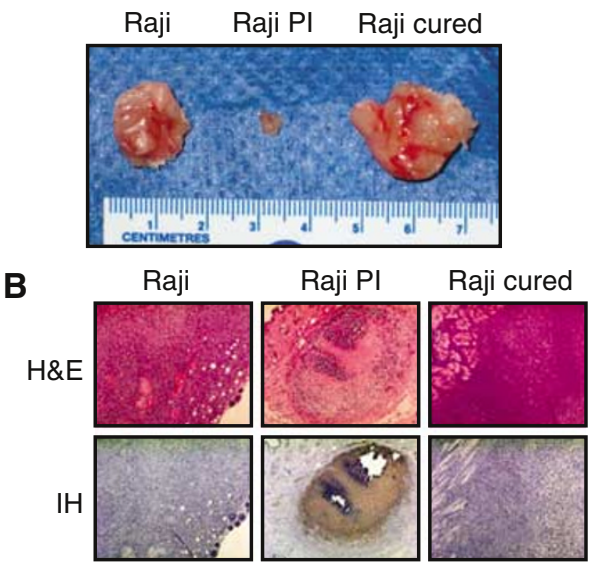

Figure 2 Tumorigenicity of Raji parental, PI and cured cells in SCID mice. (A) SCID mice were subcutaneously implanted with $1 \times 10^{7}$ cells of Raji parental, $\mathrm{Pl}$ or cured cells. Tumour growth was followed for a period of 5 weeks and two-dimensional measurements were taken weekly with a caliper. Resected tumours were photographed. (B) Haematoxylin and eosin staining and $\mathrm{IH}$ of reovirus antigens in tumour tissue taken from Raji parental, PI and cured tumours. Haematoxylin- and eosin-stained section (original magnification $\times 200$ ) show large tumours 30 days postinjection in the Raji and cured group. Tumours in the PI group were not apparent on palpation and only small foci of tumour were found on histological examination. Immunohistochemical of tumour sections (original magnification $\times 200$ ) shows that only PI cells stain positively for reovirus proteins (brown), although most of the tumour is necrotic. abilities of the three cell types to form tumours correlated with their Ras-GTP and ERK1/2 levels.

\section{Raji cured cells are resistant to reovirus oncolysis in vitro but are susceptible in vivo}

To determine the permissiveness of the Raji cured cells to reovirus infection in vitro, the cells were infected with reovirus at an MOI of $20 \mathrm{PFU} \mathrm{cell}^{-1}$ and assessed for cytopathic effects, viability, viral protein synthesis, and progeny virus production. As reported previously, Raji parental cells became granular and irregularshaped, and manifested extensive cell clumping characteristic of cytopathic effects following reovirus infection. Cured and PI cells were however resistant to reovirus even at $96 \mathrm{~h}$ postinfection. Based on WST assay performed on the cells at this time point, viability was reduced in the parental cell line to approximately $45 \%$, whereas no change in viability was detected in the cured or PI cells (Figure 3A).
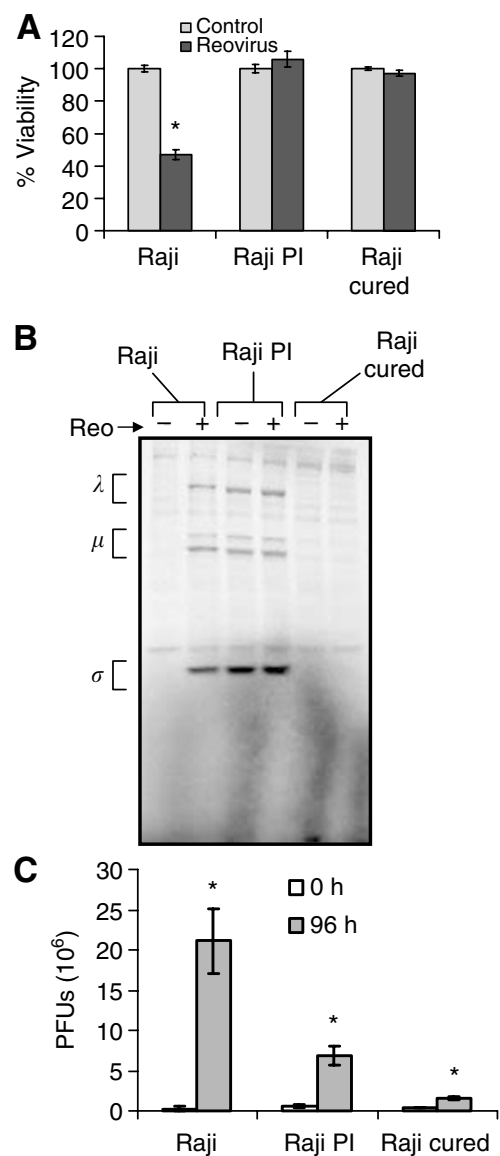

Figure 3 Effect of reovirus on Raji parental and cured cells in vitro. (A) Cell viability. Raji parental, $\mathrm{PI}$ and cured cells were exposed to reovirus at an $\mathrm{MOI}$ of $20 \mathrm{PFU} \mathrm{cell}^{-1}$, and viability at $96 \mathrm{~h}$ postinfection was assessed using WST-I assay from three independent wells. Compared to controls, $P$-values for reovirus-treated groups are $P<0.001, P=0.93$ and $P=0.222$ for Raji, Raji PI and Raji cured, respectively. (B) Viral protein synthesis. Infected cells were metabolically labelled with $\left.{ }^{35} \mathrm{~S}\right]$ methionine for $6 \mathrm{~h}$ at $42 \mathrm{~h}$ postinfection. Cell lysates were prepared, and reovirus proteins were immunoprecipitated with a polyclonal anti-reovirus antibody and analysed by SDS-PAGE. The three size classes of reovirus proteins $(\lambda, \mu$ and $\sigma)$ are indicated on the left. (C) Virus progeny. At 0 and $96 \mathrm{~h}$ postinfection, cells were harvested and freeze-thawed three times, and the virus titre in the lysate was determined by plaque assay on $L 929$ cells. Compared to input virus, $P$-values for progeny virus at $96 \mathrm{~h}$ postinfection are $P<0.00 \mathrm{I}$, $P=0.0015$ and $P<0.001$. Error bars indicate s.e.m. from three separate wells and ${ }^{*}$ demonstrates statistical significance. 
The infected cells were also metabolically labelled with $\left[{ }^{35} \mathrm{~S}\right]$ methionine, followed by immunoprecipitation with antireovirus antibody for the detection of viral protein synthesis (Figure 3B). The synthesis of the three viral protein groups $(\lambda, \mu$ and $\sigma)$ was observed in the parental Raji cells, but not in the Raji cured cells. Resistance of the cured cells to reovirus infection was also evident from the lack of progeny virus production even at $96 \mathrm{~h}$ postinfection compared to the parental cells (Figure 3C). As expected, the PI cells supported endogenous viral protein synthesis, which did not appear to be enhanced by the addition of exogenous reovirus (Figure $3 \mathrm{~B}$ ). The continuous production of endogenous virus in PI cells was accountable for the moderate enhancement in virus yield with time (Figure 3C).

In view of the aggressiveness of the cured tumours and their resistance to reovirus in vitro, we next assessed the susceptibility of these cells to reovirus treatment in vivo. To this end, parental and cured Raji lymphoma tumours grown subcutaneously in SCID mice were subjected to a single intratumoral injection of $1 \times 10^{7}$ PFUs of reovirus. We were surprised to find that, contrary to their behaviour in vitro, Raji cured cells were now highly susceptible to reovirus in vivo (Figure 4A). To determine if cell death occurred by active viral infection, immunohistochemical (IH) staining with anti-reovirus antibody was performed on paraffin-embedded sections collected 24 days after reovirus injection (Figure 4B). Haematoxylin/eosin staining confirmed the

A

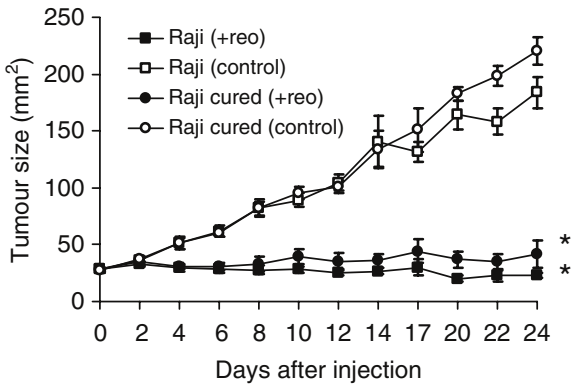

B

Raji
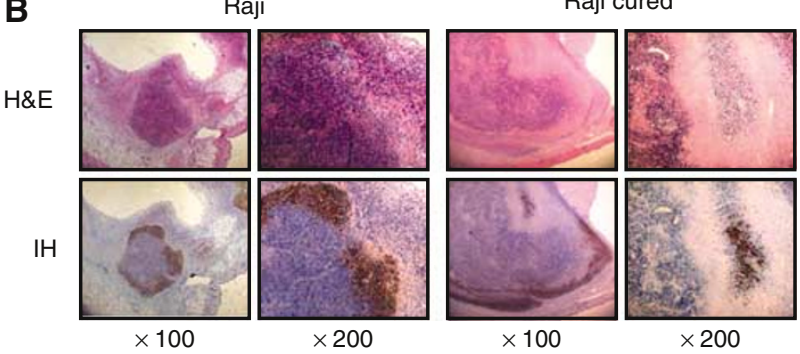

Figure 4 Effect of reovirus on parental and cured Raji tumours in vivo. (A) Intratumoral reovirus therapy of lymphoid tumours in SCID mice. SCID mice were subcutaneously implanted with $1 \times 10^{7}$ cells of either Raji parental or cured cells. Following palpable tumour establishment, the tumours received (on day 0 ) a single intratumoral injection of $1 \times 10^{7}$ PFUs of live reovirus $(n=8)$ or saline control $(n=7)$. Tumour growth was followed for a period of 25 days and measured two-dimensionally with a caliper. *Differences are statistically significant with $P<0.001$ for Raji + reovirus and $P=0.002$ for Raji cured + reovirus compared to respective controls. (B) Haematoxylin and eosin staining and $\mathrm{H}$ of reovirus antigens in Raji parental and cured tumours after intratumoral reovirus treatment. Haematoxylin- and eosin-stained section shows necrosis of tumour cells 24 days after live reovirus treatment. $\|$-stained section (original magnification $\times 100$ (left) and $\times 200$ (right)) of remaining tumour cells stains positively for reovirus proteins (brown). Histological examination of saline-treated (control) Raji and cured tumours showed very large actively proliferating tumours (data not shown). killing of tumour cells in the live virus-treated group, and $\mathrm{IH}$ staining with anti-reovirus antibody showed the presence of viral proteins in the residual tumour cells of both Raji parental and Raji cured cells. Therefore, cured cells that are resistant to reovirus in vitro become susceptible to reovirus in vivo.

\section{Cured cells are susceptible to in vitro infection by protease-stripped virions}

Based on current information on reovirus infection strategy, we speculated that the discrepancy between the in vitro and in vivo data could be due to the proteolytic microenvironment within tumours that enhanced reovirus infection of the cured tumours by converting reovirions into ISVPs. Such ISVPs have been shown to be capable of infecting many restrictive cells that have somehow lost their ability to process the uncoating of the incoming virion (Dermody et al, 1993; Baer and Dermody, 1997; Wetzel et al, 1997; Baer et al, 1999; Golden et al, 2002).

To test this idea, we first determined if resistance of the cured cells to reovirus killing in vitro could be reversed by the inclusion of a protease during infection with intact reovirions or by using protease-stripped virions (ISVPs) directly. The results (Figure 5A) show that there was a significant increase in cell death when infection by reovirions was carried out in the presence of chymotrypsin, or when ISVPs were used instead of reovirions. Infected cells were also labelled with $\left[{ }^{35} \mathrm{~S}\right]$ methionine, followed by immunoprecipitation with anti-reovirus antibody for the detection of viral protein synthesis (Figure 5B). A drastic increase in synthesis of the three viral protein groups $\lambda, \mu$ and $\sigma$, was evident in the Raji cured cells following infection with reovirions in the presence of chymotrypsin or with ISVPs alone. WST-1 assay confirmed the loss in viability of the cured cells from these treatments (Figure 5C). These results clearly show that the resistance of the cured cells to infection with reovirus can be overcome by proteolytic processing of reovirus into ISVP.

\section{Protease inhibitor E64 prevents reovirus oncolysis in vitro and in vivo}

To see if a similar protease processing of virions occurs within Raji cured tumours, which results in their susceptibility to killing by reovirions in vivo, we used the specific cysteine protease inhibitor E64, which has been shown to block the conversion of reovirions to ISVPs (Baer and Dermody, 1997; Chandran and Nibert, 1998). We first tested the effect of E64 on Raji and Raji cured cells in vitro. The results (Figure 6A) show that E64 effectively blocked infection of the Raji cells by reovirus but not by ISVPs. Raji cured cells were once again only efficiently infected by ISVP and in the presence of E64 as well.

If indeed a proteolytic environment in tumours is important for reovirus permissiveness in vivo, as it is for cells in vitro, treating mice bearing tumours with a protease inhibitor should also impair reovirus oncolysis in vivo. To test this, mice bearing subcutaneous Raji tumours were injected intraperitoneally (i.p.) with E64 before a single intratumoral reovirus injection. I.p. administration of E64 was continued every 2 days until day 8. As observed above, reovirus treatment alone effectively prevented tumour progression; however, reovirus therapy was rendered ineffective in E64-treated mice (Figure 6B). Furthermore, upon termination of the E64 treatment (at day 8), the tumours were seen to regress. These results suggest that the microenvironment inside the cured tumours is proteolytic, and such a proteolytic environment promotes the conversion of reovirions to ISVPs, which can then infect and kill the cured tumour cells that have been selected for their resistance to infection by intact virions. 

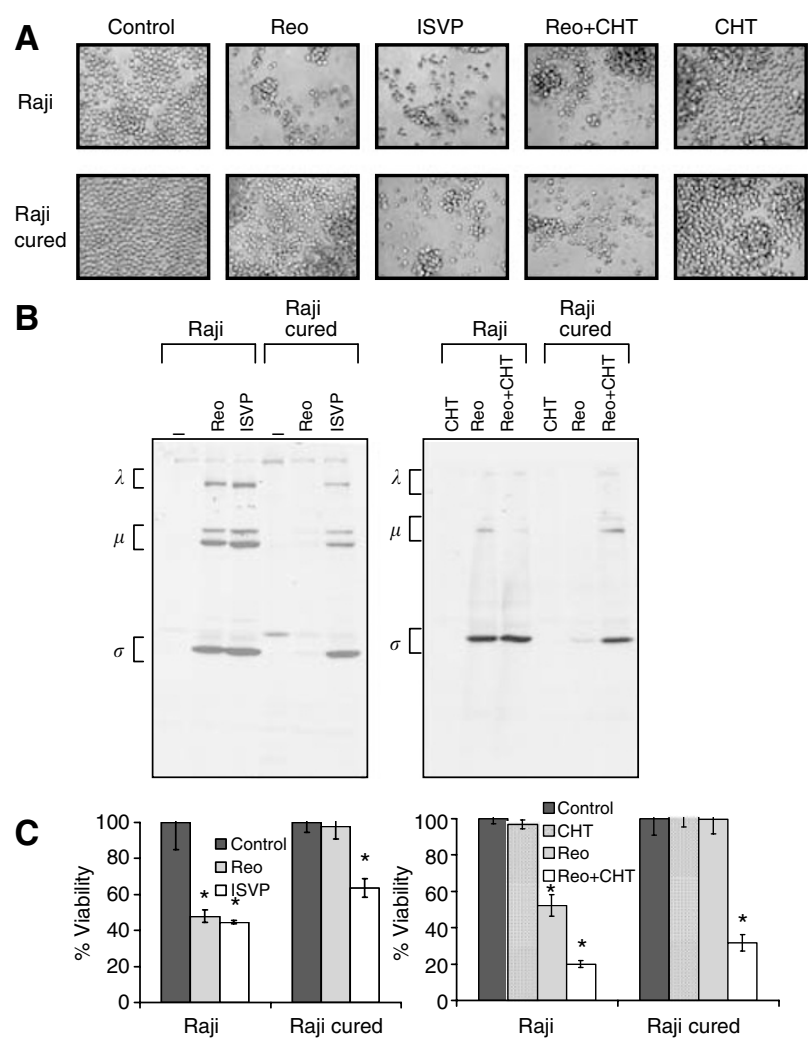

Figure 5 Infection of Raji cured cells by ISVP, or by reovirions in the presence of chymotrypsin. (A) Cytopathic effects. Raji parental or cured cells were exposed to ISVP, reovirus or reovirus in the presence of chymotrypsin (CHT) at an $\mathrm{MOI}$ of $20 \mathrm{PFU} \mathrm{cell}^{-1}$, and photomicrographs were taken at $72 \mathrm{~h}$ postinfection. Original magnification $\times 400$. (B) Reovirus protein synthesis in infected Raji parental and cured cells. Cells were infected with ISVP, reovirus or reovirus in the presence of $\mathrm{CHT}$, and pulse-labelled with $\left[{ }^{35} \mathrm{~S}\right]$ methionine for $6 \mathrm{~h}$ at $18 \mathrm{~h}$ postinfection. The cells were then harvested and lysed, and reovirus proteins were immunoprecipitated from an aliquot of the lysate using a rabbit polyclonal anti-reovirus antibody, followed by SDS-PAGE. The three size classes of reovirus proteins $(\lambda, \mu$ and $\sigma)$ are indicated on the left. (C) Viability of Raji parental and cured cells infected with ISVP, reovirus or reovirus in the presence of CHT. WST-I assay was carried out on cells from three independent wells at $96 \mathrm{~h}$ postinfection. P-values compared with respective uninfected controls are: Left panel, $P=0.012$ (Raji + Reo), $P=0.017$ (Raji + ISVP), $P=0.6 I$ (Raji cured + Reo) and $P=0.026$ (Raji cured + ISVP). Right panel, $P=0.019 \quad$ (Raji + Reo), $P<0.001 \quad$ (Raji + Reo $+C H T$ ),$P=0.98$ (Raji cured + Reo) and $P=0.0013$ (Raji cured + Reo $+\mathrm{CHT}$ ). * Indicates statistical significance.

\section{DISCUSSION}

Resistance to reovirus oncolysis is a phenomenon previously only known to occur in vitro for a variety of cell types. This occurs through the acquisition of a PI or cured cellular state (Taber et al, 1976; Ahmed et al, 1981; Ahmed and Fields, 1982; Danis et al, 1993; Dermody et al, 1993, 1995; Baer and Dermody, 1997; Wetzel et al, 1997; Chappell et al, 1998; Baer et al, 1999; Ebert et al, 2004). The aim of this study was to evaluate the potential limitations that the emergence of PI or cured cells may pose for reovirus oncolytic therapy. To assess this, we investigated the tumorigenicity and subsequent susceptibility to reovirus of a PI Burkitt lymphoma cell line (Raji) and its respective cured and parental counterparts. Our data suggest that these two cellular states are not likely to be a significant barrier to reovirus oncolysis clinically for two reasons: (1) PI Raji cells were not tumorigenic in SCID mice, and (2) while cured Raji cells readily form tumours, they were efficiently

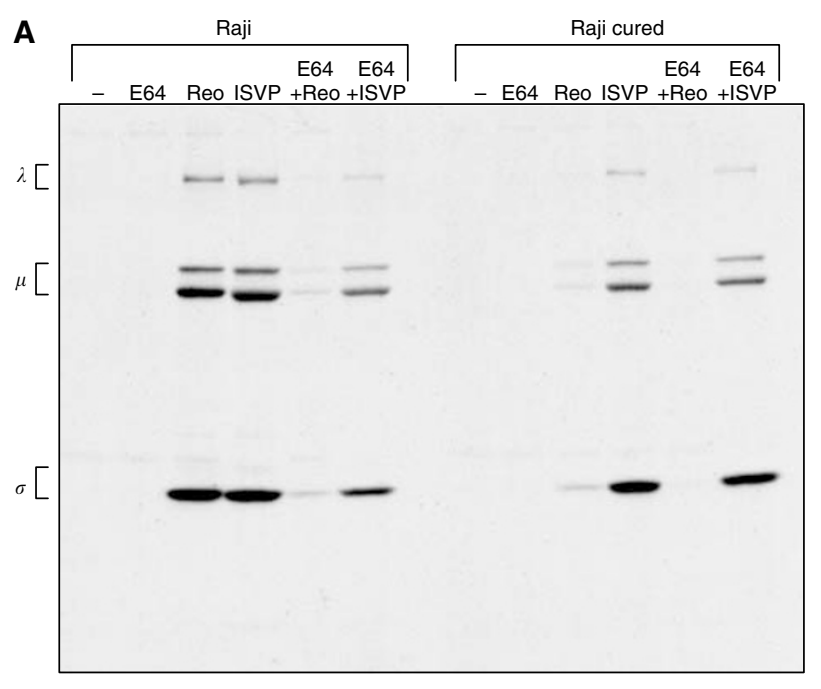

B

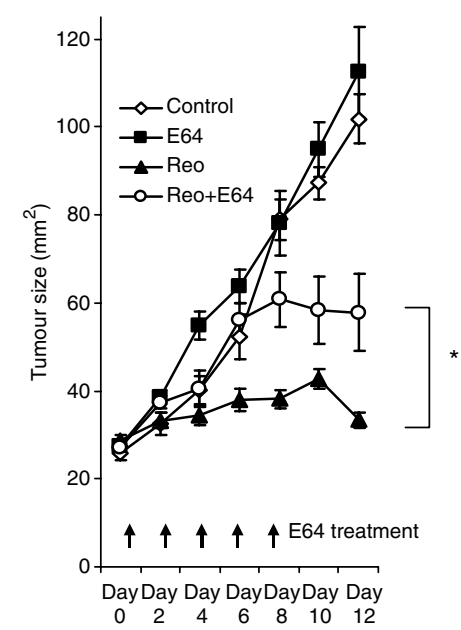

Figure 6 Infection of Raji and Raji cured cells in the presence of E64. (A) Reovirus protein synthesis in infected Raji parental and cured cells treated with E64. Cells were infected with ISVP or reovirus in the presence or absence of E64, and pulse-labelled with $\left[{ }^{35}\right.$ S $]$ methionine for $6 \mathrm{~h}$ at $18 \mathrm{~h}$ postinfection. The cells were then harvested and lysed, and reovirus proteins were immunoprecipitated from an aliquot of the lysate using a rabbit polyclonal anti-reovirus antibody, followed by SDS-PAGE. The three size classes of reovirus proteins $(\lambda, \mu$ and $\sigma)$ are indicated on the left. (B) Intratumoral reovirus therapy of lymphoid tumours in SCID mice exposed to E64 injected i.p. SCID mice were subcutaneously implanted with $1 \times 10^{7}$ cells of Raji parental cells. Following palpable tumour establishment, the tumours received (on day 0 ) either I mg per mouse of E64 in PBS alone $(n=5)$ or I h before a single intratumoral injection of $\mathrm{I} \times 10^{7}$ PFUs of live reovirus $(n=5)$ and $\mathrm{E} 64$ was re-injected subsequently every second day until day 8. E64 treatments were compared to PBS control and reovirus alone group $(n=5)$. Tumour growth was followed for a period of 12 days and measured two-dimensionally with a caliper. *Wilcoxon's signed-rank test $(P=0.0379)$ comparing tumour sizes of reovirus + E64-treated group to reovirus-alone group.

eliminated by a single injection of reovirus despite their complete resistance to oncolysis in vitro. This latter observation indicates that reovirus' oncolytic properties may be enhanced in vivo and that in vitro testing of cell lines may not predict in vivo susceptibility.

Reovirus is known to produce a persistent infection in a wide range of cell lines including cancer cells (Ahmed et al, 1981; Ahmed and Fields, 1982; Dermody et al, 1993; Dermody, 1998). Despite this well-described cellular state, this is the first report examining the tumorigenicity of reovirus PI or cured cancer cells. 
Our present observation that reovirus PI cells regain their tumorigenicity upon clearance of the virus suggests that these PI cells have never lost their intrinsic tumorigenic potential. Rather, this potential is somehow suppressed by the presence of the resident virus. In this regard, it is interesting to note that reovirus PI 3T3 cells reportedly exhibited a decrease of $70-90 \%$ in the number of the epidermal growth factor (EGF) receptors and a corresponding decrease in EGF-stimulated DNA synthesis (Verdin et al, 1986). Similarly, we observed a marked reduction of activated Ras-GTP and phosphorylated ERK1/2 kinases in the PI cells when compared to parental and cured cells. Reduced expression or activation of important oncogenes and/or growth factors due to the chronic presence of viral proteins and/or transcripts may explain why these cells fail to colonise in soft agar and to form tumours in mice. It is also noteworthy that persistent infection of cancer lines by other oncolytic viruses, such as vesicular stomatis virus (VSV), measles, adenovirus or herpesvirus, also occurs (Minato et al, 1979; Wolfson et al, 1991; Flomenberg et al, 1996; Kao et al, 1999), and that measles and VSV PI cells were reportedly incapable of forming tumours in vivo (Minato et al, 1979; Schattner et al, 1985). Similar to the reovirus PI cells reported by Verdin et al, measles PI neuroblastoma cells showed a markedly depressed level of H-Ras expression, which correlated with the loss of malignant phenotypes (Wolfson et al, 1991). Whether these cells can regain their tumorigenicity upon viral clearance is unknown at present.

Our studies on the Raji cured cells have led us to suggest that proteases present within these tumours are responsible for the enhanced reovirus infection in vivo, since these cells are only efficiently infected in vitro when reovirus is first proteolytically processed into ISVP before infection. Indeed, addition of exogenous proteases to the culture medium has been shown to facilitate reovirus infection of a variety of cells, which would not otherwise be susceptible (Golden et al, 2002). Studies have shown that protease-generated ISVPs are capable of directly penetrating the cell membrane and entering the cytoplasm where viral replication can take place (Borsa et al, 1979; Chandran et al, 2002). Such a scenario probably plays out in the human gastrointestinal tract where the presence of intestinal proteases likely promotes reovirus infection. It is noteworthy that proteolytic conversion of reovirions into ISVPs in the intestinal lumen has been shown to be essential for efficient reovirus infection in mice
(Amerongen et al, 1994), and inhibitors of luminal serine proteases reduce viral infectivity (Bass et al, 1990). Inhibitors of endosomal and lysosomal proteases have been shown to restrict reovirus infection in cell culture (Dermody et al, 1993; Baer and Dermody, 1997; Wetzel et al, 1997; Chandran and Nibert, 1998; Baer et al, 1999). In this study, we observed that addition of the protease inhibitor E64 blocks reovirus oncolysis of Raji cells in vitro but importantly also impairs Raji tumour regression in vivo, suggesting the importance of proteases in reovirus oncolysis. Since high levels of protease activity are known to exist in the microenvironment of many tumours, particularly at the tumour-stroma interface, and increased protease production occurs in a variety of transformed cells (Rubin, 2003), tumours present an ideal environment for reovirus replication. Furthermore, cells transformed with oncogenic Ras have increased expression of proteases (Chambers et al, 1992), and we have shown that reovirus selectively infects cells with an activated Ras pathway (Coffey et al, 1998; Strong et al, 1998). Collectively, our data suggest that the proteolytic environment in tumours probably facilitates reovirus uncoating, and this could account for its enhanced oncolytic capability in vivo.

In view of the reovirus clinical trials currently underway, results from this study should alleviate some concerns regarding the potential problems with the tumorigenic potential of PI tumour cells, as well as with the resistance of the cured tumour cells to reovirus therapy. These results further suggest that reovirus could have broadened oncolytic capabilities due to enhanced proteolytic processing of virions within tumours, and that in vitro testing of cancer cells or tumour specimens does not necessarily predict which tumours will be resistant to reovirus infection in vivo.

\section{ACKNOWLEDGEMENTS}

This work was supported by grants from the Canadian Institute of Health Research (to PWKL), the National Cancer Institute of Canada with funds from the Canadian Cancer Society (to PF), the Alberta Cancer Board (No. R1-170) and Leukemia Research Fund of Canada - The United Food and Commercial Workers Union Award (to AEK). TA is supported by the Canadian Institute for Health Research and by the Alberta Heritage Foundation for Medical Research.

\section{REFERENCES}

Ahmed R, Fields BN (1982) Role of the S4 gene in the establishment of persistent reovirus infection in L cells. Cell 28: 605-612

Ahmed R, Canning WM, Kauffman RS, Sharpe AH, Hallum JV, Fields BN (1981) Role of the host cell in persistent viral infection: coevolution of $\mathrm{L}$ cells and reovirus during persistent infection. Cell 25: 325-332

Alain T, Hirasawa K, Pon KJ, Nishikawa SG, Urbanski SJ, Auer Y, Luider J, Martin A, Johnston RN, Janowska-Wieczorek A, Lee PW, Kossakowska AE (2002) Reovirus therapy of lymphoid malignancies. Blood 100: 41464153

Amerongen HM, Wilson GA, Fields BN, Neutra MR (1994) Proteolytic processing of reovirus is required for adherence to intestinal $M$ cells. J Virol 68: $8428-8432$

Baer GS, Dermody TS (1997) Mutations in reovirus outer-capsid protein sigma3 selected during persistent infections of $\mathrm{L}$ cells confer resistance to protease inhibitor E64. J Virol 71: 4921-4928

Baer GS, Ebert DH, Chung CJ, Erickson AH, Dermody TS (1999) Mutant cells selected during persistent reovirus infection do not express mature cathepsin L and do not support reovirus disassembly. J Virol 73: 95329543

Bass DM, Bodkin D, Dambrauskas R, Trier JS, Fields BN, Wolf JL (1990) Intraluminal proteolytic activation plays an important role in replication of type 1 reovirus in the intestines of neonatal mice. $J$ Virol 64: $1830-1833$

Bell JC, Lichty B, Stojdl D (2003) Getting oncolytic virus therapies off the ground. Cancer Cell 4: 7-11

Borsa J, Morash BD, Sargent MD, Copps TP, Lievaart PA, Szekely JG (1979) Two modes of entry of reovirus particles into L cells. J Gen Virol 45: $161-170$

Chambers AF, Colella R, Denhardt DT, Wilson SM (1992) Increased expression of cathepsins L and B and decreased activity of their inhibitors in metastatic, ras-transformed NIH 3T3 cells. Mol Carcinogen 5: $238-245$

Chandran K, Nibert ML (1998) Protease cleavage of reovirus capsid protein $\mathrm{mu} 1 / \mathrm{mulC}$ is blocked by alkyl sulfate detergents, yielding a new type of infectious subvirion particle. J Virol 72: 467-475

Chandran K, Farsetta DL, Nibert ML (2002) Strategy for nonenveloped virus entry: a hydrophobic conformer of the reovirus membrane penetration protein micro 1 mediates membrane disruption. $J$ Virol 76: $9920-9933$

Chappell JD, Barton ES, Smith TH, Baer GS, Duong DT, Nibert ML, Dermody TS (1998) Cleavage susceptibility of reovirus attachment protein sigmal during proteolytic disassembly of virions is determined by a sequence polymorphism in the sigmal neck. J Virol 72: $8205-8213$

Coffey MC, Strong JE, Forsyth PA, Lee PW (1998) Reovirus therapy of tumors with activated Ras pathway. Science 282: $1332-1334$ 
Danis C, Mabrouk T, Garzon S, Lemay G (1993) Establishment of persistent reovirus infection in $\mathrm{SC} 1$ cells: absence of protein synthesis inhibition and increased level of double-stranded RNA-activated protein kinase. Virus Res 27: 253-265

Dermody TS (1998) Molecular mechanisms of persistent infection by reovirus. Curr Top Microbiol Immunol 233: 1-22

Dermody TS, Chappell JD, Hofler JG, Kramp W, Tyler KL (1995) Eradication of persistent reovirus infection from a B-cell hybridoma. Virology 212: 272-276

Dermody TS, Nibert ML, Wetzel JD, Tong X, Fields BN (1993) Cells and viruses with mutations affecting viral entry are selected during persistent infections of L cells with mammalian reoviruses. J Virol 67: 2055-2063

Ebert DH, Kopecky-Bromberg SA, Dermody TS (2004) Cathepsin B is inhibited in mutant cells selected during persistent reovirus infection. J Biol Chem 279: 3837-3851

Etoh T, Himeno Y, Matsumoto T, Aramaki M, Kawano K, Nishizono A, Kitano S (2003) Oncolytic viral therapy for human pancreatic cancer cells by reovirus. Clin Cancer Res 9: 1218-1223

Flomenberg P, Piaskowski V, Harb J, Segura A, Casper JT (1996) Spontaneous, persistent infection of a B-cell lymphoma with adenovirus. J Med Virol 48: 267-272

Golden JW, Linke J, Schmechel S, Thoemke K, Schiff LA (2002) Addition of exogenous protease facilitates reovirus infection in many restrictive cells. $J$ Virol 76: $7430-7443$

Hirasawa K, Nishikawa SG, Norman KL, Alain T, Kossakowska A, Lee PW (2002) Oncolytic reovirus against ovarian and colon cancer. Cancer Res 62: $1696-1701$

Kao YS, Sundin DR, Gebhardt BM (1999) Persistent infection of a lymphoma cell line by herpes simplex virus. Am J Hematol 62: 93-98

Kilani RT, Tamimi Y, Hanel EG, Wong KK, Karmali S, Lee PW, Moore RB (2003) Selective reovirus killing of bladder cancer in a co-culture spheroid model. Virus Res 93: 1-12

Kirn D (2002) Virotherapy for cancer: current status, hurdles, and future directions. Cancer Gene Ther 9: 959-960

Laemmli UK (1970) Cleavage of structural proteins during the assembly of the head of bacteriophage T4. Nature 227: 680-685

Lee PW, Hayes EC, Joklik WK (1981) Characterization of anti-reovirus immunoglobulins secreted by cloned hybridoma cell lines. Virology 108: $134-146$

Minato N, Bloom BR, Jones C, Holland J, Reid LM (1979) Mechanism of rejection of virus persistently infected tumor cells by athymic nude mice. J Exp Med 149: 1117-1133
Morris DG, Forsyth PA, Paterson AH, Fonseca K, Difrancesco LM, Thompson BG, Coffey MC (2002) A phase I clinical trial evaluating intralesional Reolysin (reovirus) in histologically confirmed malignancies. ASCO Annual Meeting. Orlando, FL, USA

Norman KL, Coffey MC, Hirasawa K, Demetrick DJ, Nishikawa SG, DiFrancesco LM, Strong JE, Lee PW (2002) Reovirus oncolysis of human breast cancer. Hum Gene Ther 13: 641-652

Rubin H (2003) Microenvironmental regulation of the initiated cell. Adv Cancer Res 90: 1-62

Schattner A, Rager-Zisman B, Bloom BR (1985) Persistent viral infection affects tumorigenicity of a neuroblastoma cell line. Cell Immunol 90: $103-114$

Sebti SM, Jani JP, Mistry JS, Gorelik E, Lazo JS (1991) Metabolic inactivation: a mechanism of human tumor resistance to bleomycin. Cancer Res 51: 227-232

Smith RE, Zweerink HJ, Joklik WK (1969) Polypeptide components of virions, top component and cores of reovirus type 3. Virology 39: 791 810

Strong JE, Coffey MC, Tang D, Sabinin P, Lee PW (1998) The molecular basis of viral oncolysis: usurpation of the Ras signaling pathway by reovirus. Embo J 17: 3351-3362

Taber R, Alexander V, Whitford W (1976) Persistent reovirus infection of CHO cells resulting in virus resistance. J Virol 17: 513-524

Tyler KL, Fields BN (1996) Reoviruses. In Fields Virology Fields BN, Knipe DM, Howley PM (eds) Philadelphia, PA: Lippincott-Raven, pp 15971623

Verdin EM, Maratos-Flier E, Carpentier JL, Kahn CR (1986) Persistent infection with a nontransforming RNA virus leads to impaired growth factor receptors and response. J Cell Physiol 128: 457-465

Wetzel JD, Wilson GJ, Baer GS, Dunnigan LR, Wright JP, Tang DS, Dermody TS (1997) Reovirus variants selected during persistent infections of $\mathrm{L}$ cells contain mutations in the viral S1 and S4 genes and are altered in viral disassembly. J Virol 71: $1362-1369$

Wilcox ME, Yang W, Senger D, Rewcastle NB, Morris DG, Brasher PM, Shi ZQ, Johnston RN, Nishikawa S, Lee PW, Forsyth PA (2001) Reovirus as an oncolytic agent against experimental human malignant gliomas. J Natl Cancer Inst 93: 903-912

Wolfson M, Gopas J, Katorza A, Udem SA, Segal S, Rager-Zisman B (1991) Regulatory effects of persistent measles virus infection on tumorigenicity and protooncogene expression in neuroblastoma cells. Cancer Detect Prev 15: $171-176$ 